\title{
Characterization of Propagation Patterns With Omnipolar EGM in Epicardial Multi-Electrode Arrays
}

\author{
Jennifer Riccio ${ }^{1}$, Alejandro Alcaine ${ }^{2,1}$, Natasja MS de Groot ${ }^{3}$, Richard Houben ${ }^{4}$, Pablo Laguna ${ }^{1,2}$, \\ Juan Pablo Martínez ${ }^{1,2}$ \\ ${ }^{1}$ BSICoS, Aragon Institute of Engineering Research, IIS Aragón, Universidad de Zaragoza, \\ Zaragoza, Aragon, Spain \\ ${ }^{2}$ CIBER en Bioingeniería, Biomateriales y Nanomedicina (CIBER-BBN), Madrid, Spain \\ ${ }^{3}$ Department of Cardiology, Erasmus University Medical Center, Rotterdam, The Netherlands \\ ${ }^{4}$ 2BMedical BV, Maastricht, The Netherlands
}

\begin{abstract}
Omnipolar Electrogram (OP-EGM) is a recently proposed technique to characterize myocardial propagation in multi-electrode catheters regardless of the angle between propagation direction and catheter bipolar. This work aims to evaluate the accuracy of atrial propagation parameters obtained with OP-EGM in sinus rhythm (SR) and in different patterns of atrial fibrillation $(A F)$.

Real and simulated unipolar electrograms (u-EGMs) were used in this study. For both types of data, conduction velocity was obtained for each clique of 4 neighbour electrodes using OP-EGM. As a reference, conduction velocity was also computed from local activation times (LATs) using a linear propagation model.

Analysis of simulated data showed that conduction velocity had good concordance with propagation patterns for both estimations, although the LAT-based errors were lower in most of the cases. When conduction velocity magnitude $(\mathrm{CV})$ was $1 \mathrm{~mm} / \mathrm{ms}$, its estimation errors (expressed as mean \pm std) calculated with OP-EGM and from LATs were $0.053 \pm 0.005 \mathrm{~mm} / \mathrm{ms}$ and $0.003 \pm 2.1 \times 10^{-5}$ $\mathrm{mm} / \mathrm{ms}$, respectively, when focus was at $30 \mathrm{~mm}$ from the bottom of the tissue slice, while propagation direction angular errors were $6.64 \pm 4.3^{\circ}$ and $4.35 \pm 2.81^{\circ}$. In real data, maps obtained with OP-EGM presented smoother and more coherent patterns than those based on LATs.
\end{abstract}

\section{Introduction}

Atrial fibrillation (AF) is the most frequent cardiac arrhythmia, involving a high number of hospitalizations. It shows a significant increase with age and its consequences include heart failure, stroke, dementia and doubled mortality [1].

$\mathrm{AF}$ is a consequence of an incorrect interaction between activation mechanisms and an anomalous atrial substrate. However these mechanisms, as well as those explaining the maintenance and recurrence of AF, are not completely understood and not mutually exclusive, thus complicating the understanding and treatment of this arrhythmia.

One of the possible therapies for AF is catheter ablation. It consists on delivering radiofrequency energy using invasive catheters to burn the area of cardiac tissue involved into the AF, typically around the pulmonary veins (PV) [1]. This isolation avoids the ectopic impulses to spread from the PV to the atrium and trigger the arrhythmia. Other ablation strategies can be used together with PV isolation, in order to prevent the generation and perpetuation of AF. These strategies require analysis of intracardiac electrograms (EGMs) features in order to better understand the mechanisms responsible for $\mathrm{AF}$ and, consequently, to find the appropriate ablation site (EGM-guided ablation).

Due to the complexity and instability of AF, highdensity simultaneous EGMs are to be preferred over sequential EGM analysis. This is made possible thanks to the development of multipolar diagnostic catheters, having high spatial resolution and allowing spatial multiprocessing of the signal. Typically, unipolar EGMs (u-EGMs) and bipolar EGMs (b-EGMs) are used to calculate propagation parameters. However, the former are very much affected by far-field, while the latter are very dependent on the direction of the activation wavefront with respect to the orientation of the recording electrodes pair. Omnipolar EGM (OP-EGM) is a novel technique which overcomes the above mentioned limitations, using an estimation of the local electric field from the EGMs recorded at each clique of nearby electrodes to find propagation parameters [3].

In this work, we evaluated the accuracy of conduction velocity magnitude $(\mathrm{CV})$ and propagation direction obtained with the OP-EGM method. We also derived the same propagation parameters from detected local activa- 
tion times (LATs), using a linear model obtained in the least squares sense. These evaluations have been performed both for clinical and simulated u-EGMs.

\section{Materials and Methods}

\subsection{Simulated data}

In order to assess the accuracy of the conduction velocity estimates obtained from EGMs, a uniform doublelayer model of a planar slice of atrial tissue was used to simulate propagating patterns from a single focus. Tissue slices of $18 \times 34 \times 2 \mathrm{~mm}$ were simulated for isotropic and anisotropic tissues (with an anisotropy ratio of 0.5 , i.e. double velocity in $y$-axis than in $x$-axis or vice versa). In a rectangular $8 \times 16$ high-density multi-electrode array (MEA) centered in the 2-D tissue slice, with interelectrode distance of $2 \mathrm{~mm}$, u-EGMs were computed. For each set of tissue properties, three different foci were considered for the activation: at $30 \mathrm{~mm}$ from the bottom, at the middle and in the right inferior corner of the slice.

Each simulated u-EGM contains one activation (corresponding to one sinus beat) and was low-pass filtered with $150 \mathrm{~Hz}$ cut-off frequency. LATs were estimated as the samples where the u-EGMs present their maximum negative slope.

\subsection{Clinical data}

Epicardial u-EGMs recorded during sinus rhythm (SR) and AF with a 2-D high-density MEA sensor [2] were also used in this study. The MEA was positioned on the epicardial wall of the right atrium of a patient undergoing openchest surgery coming from the Erasmus Medical Center Rotterdam (Rotterdam, The Netherlands). The MEA sensor was composed by 128 electrodes $2 \mathrm{~mm}$-apart one from each other and organized in an 8x16 rectangular grid. Corner electrodes were not available for recording, so just 124 u-EGMs were available for this study. The u-EGMs were sampled at $1 \mathrm{kHz}$ and low-pass filtered with $150 \mathrm{~Hz}$ cut-off frequency. The recording length during SR was $5 \mathrm{~s}$ and 10 s during AF. LATs manually detected by an expert electrophysiologist during SR and AF were available for MEA placed on the right atrium. The propagation pattern in SR as well as several markedly different AF patterns were selected for conduction velocity assessment.

\subsection{Estimation of conduction velocity with OP-EGM method}

$\mathrm{CV}$ and propagation direction were calculated using the OP-EGM method, which assumes a locally plane and homogeneous wave travelling within each group of four nearby electrodes within the MEA [3], referred as rectangular clique. All the features obtained with this method are considered to be located at the center of each clique.

The OP-EGM method is based on the relationship between the spatial gradient of the unipolar voltage $\phi(t)$ and the electric field $\mathbf{E}(t)$ at the extracellular-myocardial interface: $\mathbf{E}(t)=-\nabla \phi(t)$. A 3-D right-handed omnipolar coordinate system, defined by the myocardial surface normal $\overrightarrow{\mathbf{n}}$, propagation direction $\overrightarrow{\mathbf{a}}$ and wavecrest direction $\overrightarrow{\mathbf{w}}$ of the voltage wave, is considered. Therefore, at each point of the surface, $\mathbf{E}(t)$ has got the following three components, respectively: $E_{n}(t), E_{a}(t)$ and $E_{w}(t)$. Since we are considering in this study a 2-D arrangement of electrodes, we can only estimate $\mathbf{E}(t)$ in the $\overrightarrow{\mathbf{a}}-\overrightarrow{\mathbf{w}}$ plane.

For each clique of four electrodes, six different b-EGMs can be computed. From these bipolar signals, the electric field at the center of the clique $\mathbf{E}(t)$ can be estimated using least squares. Afterwards, under the assumption of a plane wave propagation in the tissue within the clique, $E_{w}(t) \approx 0$, and the propagation direction $\overrightarrow{\mathbf{a}}$ can be estimated by finding the direction which maximizes the crosscorrelation $\rho_{\phi^{\prime}, E_{a}}$ between the first temporal derivative of the unipolar voltage $\left(\phi^{\prime}(t)=\partial \phi(t) / \partial t\right)$ and the electric field in that direction $E_{a}(t)=\mathbf{E}(t) \overrightarrow{\mathbf{a}}$, with CV theoretically being the proportionality factor between $\phi^{\prime}(t)$ and $E_{a}(t)$. In [3] and [4] CV is estimated by calculating the ratio of peak-to-peak values of $\phi^{\prime}(t)$ and $E_{a}(t)$. In this work we proposed a slightly different approach to estimate the $\mathrm{CV}$ more robustly, computing it as the ratio of standard deviations (SD) of $\phi^{\prime}(t)$ and $E_{a}(t)$.

$\mathrm{CV}$ values were discarded if the maximum $\rho_{\phi^{\prime}, E_{a}}$ was lower than 0.6, indicating that the planar wave hypothesis cannot be assumed in the tissue within that clique.

\subsection{Estimation of conduction velocity with LAT}

In order to have an alternative estimation of conduction velocity based on the detected LATs, a linear model was fitted to the activation times measured from a clique of 4 electrodes, similar to [5]:

$$
t(x, y)=a_{1}+a_{2} x+a_{3} y
$$

where $t(x, y)$ is the activation time at each electrode site $(x, y)$ and $a_{1}, a_{2}$ and $a_{3}$ are the coefficients estimated by least squares. Once coefficients are known, the components of conduction velocity vector $v_{x}$ and $v_{y}$ can be obtained by partial differentiation of $t(x, y)$ as follows:

$$
\begin{aligned}
& v_{x}=\frac{\frac{\partial t}{\partial x}}{\left(\frac{\partial t}{\partial x}\right)^{2}+\left(\frac{\partial t}{\partial y}\right)^{2}}=\frac{a_{2}}{a_{2}^{2}+a_{3}^{2}} \\
& v_{y}=\frac{\frac{\partial t}{\partial y}}{\left(\frac{\partial t}{\partial x}\right)^{2}+\left(\frac{\partial t}{\partial y}\right)^{2}}=\frac{a_{3}}{a_{2}^{2}+a_{3}^{2}}
\end{aligned}
$$




\subsection{Conduction velocity maps and valida- tion}

Maps of estimated CV and propagation directions were created for all activations in clinical as well as in simulated EGM signals, using both the OP-EGM method and the LAT-based estimation. For both methods any CV greater than $4 \mathrm{~mm} / \mathrm{ms}$ was considered an outlier and was not represented.

In the case of simulated patterns, as they were created with known focus, $\mathrm{CV}$ and anisotropy ratio, the true conduction velocity was obtained at the center of each clique. This allowed to compute the estimation errors for $\mathrm{CV}$ $\left(\epsilon_{C V}^{O P}, \epsilon_{C V}^{L A T}\right)$ and for propagation direction $\left(\epsilon_{\theta}^{O P}, \epsilon_{\theta}^{L A T}\right)$, obtained with both methods and for each propagation pattern (expressed as mean \pm SD across all the cliques in the MEA). Finally, mean errors were calculated above all the simulated propagation patterns for both methods.

\section{Results}

\subsection{Analysis of simulated data}

Table 1 shows the errors for $\mathrm{CV}$ and propagation directions estimated by the two considered methods with different simulation parameters. It can be observed that OPEGM presents errors slightly greater than the LAT-based estimation in most of the cases.

\subsection{Analysis of clinical data}

Figure 1 shows (from left to right) the conduction velocity maps estimated with OP-EGM and from LATs, together with the manually annotated reference LAT maps for some selected activation patterns. In particular, Figure 1(a) shows one representative activation interval during SR, whereas Figure 1(b), (c) and (d) show different patterns observed during AF.

Each conduction velocity map consists of $\mathrm{CV}$ values and propagation directions. For each clique, the $\mathrm{CV}$ value was color-coded from blue (lowest) to red (highest), whereas its respective propagation direction is plotted as an arrow whose length is proportional to CV value. When an outlier was obtained (typically due to the non-accomplishment of the planar wave hypothesis), no arrow was plotted for that clique.

As a reference, LAT maps were also color-coded from red (earliest) to blue (latest) in $10 \mathrm{~ms}$ isochrones. When a LAT could not be associated to an electrode within the MEA, a cross appeared in that location [2].
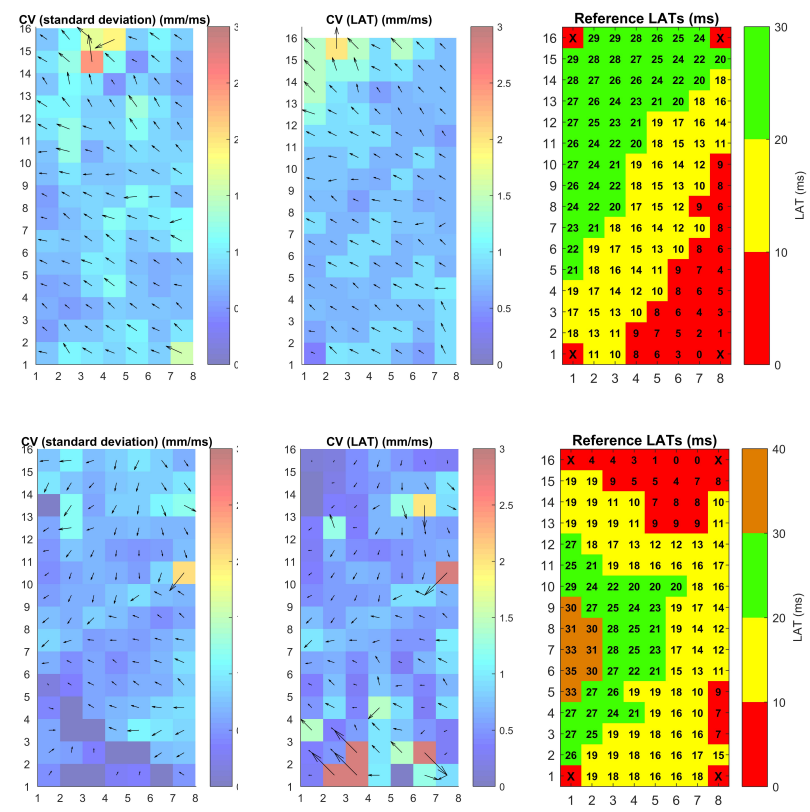

(a)
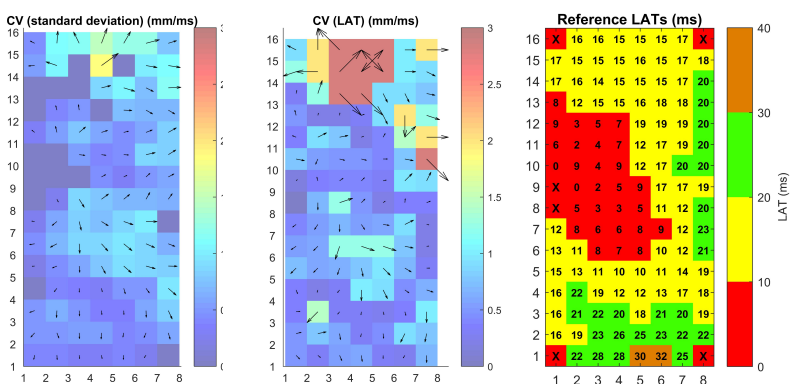

(b)
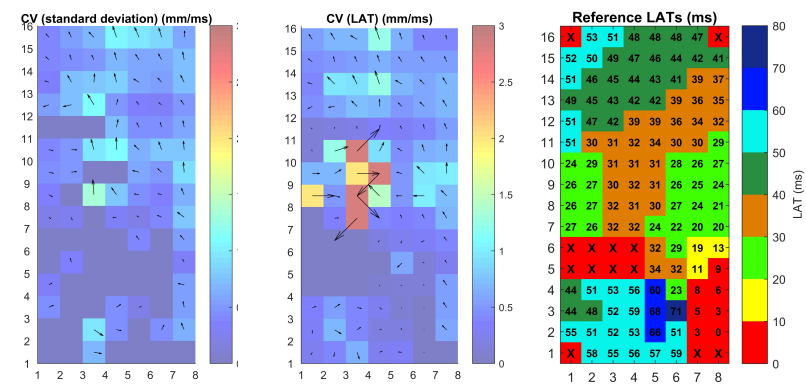

(c)

(d)

Figure 1. Maps of conduction velocities estimated with OP-EGM and with LAT-based method and reference LAT maps observed in SR (a) and during some representative propagation patterns in AF: two different wavefronts colliding and fusing into one (b), a concentric wavefront whose focus is located within the MEA (c) and a chaotic wavefront with several lines of block (d). 


\begin{tabular}{l|c|c|c|c|c} 
Simulated tissue characteristics & focus & $\epsilon_{C V}^{O P}(\mathrm{~mm} / \mathrm{ms})$ & $\epsilon_{C V}^{L A T}(\mathrm{~mm} / \mathrm{ms})$ & $\epsilon_{\theta}^{O P}(\mathrm{deg})$ & $\epsilon_{\theta}^{L A T}(\mathrm{deg})$ \\
\hline \hline \multirow{2}{*}{$\begin{array}{l}\text { Isotropic, } \\
v_{x}=v_{y}=0.6 \mathrm{~mm} / \mathrm{ms}\end{array}$} & center & $0.155 \pm 0.067$ & $0.015 \pm 0.057$ & $4.41 \pm 3.9$ & $2.35 \pm 2.69$ \\
\cline { 2 - 6 } & bottom & $0.121 \pm 0.074$ & $0.009 \pm 0.062$ & $3.78 \pm 3.21$ & $3.15 \pm 2.86$ \\
\cline { 2 - 6 } & corner & $0.177 \pm 0.071$ & $0.006 \pm 0.057$ & $7.28 \pm 5.04$ & $4.47 \pm 3.27$ \\
\hline \multirow{2}{*}{$\begin{array}{l}\text { Isotropic, } \\
v_{x}=v_{y}=1 \mathrm{~mm} / \mathrm{ms}\end{array}$} & center & $0.095 \pm 0.322$ & $0.045 \pm 0.191$ & $11.5 \pm 11.8$ & $6.99 \pm 6.42$ \\
\cline { 2 - 6 } & bottom & $0.053 \pm 0.005$ & $0.003 \pm 2.11 \times 10^{-5}$ & $6.64 \pm 4.3$ & $4.35 \pm 2.81$ \\
\cline { 2 - 6 } & corner & $0.109 \pm 0.257$ & $0.016 \pm 0.168$ & $12 \pm 8.42$ & $7.56 \pm 5.1$ \\
\hline Anisotropic, & center & $0.104 \pm 0.173$ & $0.013 \pm 0.133$ & $4.07 \pm 3.32$ & $3.95 \pm 2.64$ \\
\cline { 2 - 6 }$v_{x}=1.2 \mathrm{~mm} / \mathrm{ms}$ & bottom & $0.117 \pm 0.095$ & $0.017 \pm 0.075$ & $1.43 \pm 1.32$ & $1.49 \pm 2.35$ \\
\cline { 2 - 6 }$v_{y}=0.6 \mathrm{~mm} / \mathrm{ms}$ & corner & $0.118 \pm 0.083$ & $0.012 \pm 0.071$ & $6.47 \pm 4.81$ & $5.16 \pm 3.5$ \\
\hline Anisotropic, & center & $0.143 \pm 0.351$ & $0.051 \pm 0.222$ & $6.3 \pm 6.7$ & $4.53 \pm 3.61$ \\
\cline { 2 - 6 }$v_{x}=0.6 \mathrm{~mm} / \mathrm{ms}$ & bottom & $0.127 \pm 0.067$ & $0.012 \pm 0.065$ & $2.41 \pm 1.95$ & $3.04 \pm 2.12$ \\
\cline { 2 - 6 }$v_{y}=1.2 \mathrm{~mm} / \mathrm{ms}$ & corner & $0.154 \pm 0.174$ & $0.017 \pm 0.124$ & $8.19 \pm 6.82$ & $5.16 \pm 4.13$ \\
\hline $\begin{array}{l}\text { Anisotropic, } \\
v_{x}=2 \mathrm{~mm} / \mathrm{ms}\end{array}$ & center & $-0.049 \pm 0.149$ & $-0.019 \pm 0.069$ & $8.25 \pm 6.13$ & $5.67 \pm 3.84$ \\
\cline { 2 - 6 }$v_{y}=1 \mathrm{~mm} / \mathrm{ms}$ & bottom & $0.056 \pm 0.003$ & $0.002 \pm 7.710^{-4}$ & $1.66 \pm 0.974$ & $1.09 \pm 0.688$ \\
\cline { 2 - 6 } & corner & $0.008 \pm 0.187$ & $-0.005 \pm 0.11$ & $11.8 \pm 10.1$ & $7.68 \pm 6.59$ \\
\hline $\begin{array}{l}\text { Anisotropic, } \\
v_{x}=1 \mathrm{~mm} / \mathrm{ms}\end{array}$ & center & $-0.15 \pm 0.253$ & $-0.04 \pm 0.141$ & $11.7 \pm 9$ & $7.28 \pm 52.7$ \\
\cline { 2 - 6 }$v_{y}=2 \mathrm{~mm} / \mathrm{ms}$ & bottom & $0.051 \pm 0.008$ & $8.28 \times 10^{-4} \pm 0.004$ & $4.24 \pm 2.52$ & $2.75 \pm 1.66$ \\
\cline { 2 - 6 } & corner & $0.035 \pm 0.382$ & $0.027 \pm 0.265$ & $19.7 \pm 21.3$ & $10.3 \pm 8.25$ \\
\hline \hline
\end{tabular}

Table 1. $\quad \mathrm{CV}$ and propagation direction angular errors (presented as mean $\pm \mathrm{SD}$ ) calculated with the two considered methods for different simulated propagation patterns

\section{Discussion and conclusion}

Conduction velocity estimated from LATs and with OPEGM presented low errors both in magnitude and in propagation direction when assessed in simulated data, with the LAT-based estimation having lower errors than OPEGM. It must be noted, however, that very simple propagation patterns were simulated and that LATs were not affected by detection errors. When assessed in clinical data, LAT-based maps showed a more incoherent behavior than OP-EGM maps, especially in AF patterns. This can be explained with the fact that more complex patterns were present in these signals. Also, LAT-based maps in the clinical data were based on manually annotated LATs, which are a source of error affecting conduction velocity.

On the other side, OP-EGM maps show smoother and more coherent patterns both in SR and in the studied AF patterns, having a good concordance with LAT maps. This can be due to the OP-EGM independence from LATs, whose annotation errors do not affect conduction velocity.

\section{Acknowledgements}

This work is part of a project that has received funding from the European Union's Horizon 2020 research and innovation program under the Marie Skłodowska-Curie grant agreement No 766082 (My-ATRIA).

This work was also supported by projects DPI201675458-R (MINECO) and Aragón Government (Reference
Group BSICoS T39-17R) cofunded by FEDER 2014-2020 "Building Europe from Aragon".

\section{References}

[1] Calkins $\mathrm{H}$ et al. 2017 HRS/EHRA/ECAS/APHRS/ SOLAECE expert consensus statement on catheter and surgical ablation of atrial fibrillation. Europace 2018; 20(1):e1e160.

[2] Alcaine A et al. Spatiotemporal model-based estimation of high-density atrial fibrillation activation maps. Digital Signal Processing 2016; 54:64-74.

[3] Deno DC et al. Orientation-independent catheter-based characterization of myocardial activation. IEEE Trans Biomed Eng. 2017; 64(5):1067-1077.

[4] Massé S et al. Resolving myocardial activation with novel omnipolar electrograms. Circ Arrhythm Electrophysiol. 2016; 9:e004107.

[5] Bayly PV et al. Estimation of conduction velocity vector fields from epicardial mapping data. IEEE Trans Biomed Eng. 1998; 45(5):563-571.

Address for correspondence:

Jennifer Riccio

C/ Mariano Esquillor s/n, Edificio I+D+i, Lab 4.0.05

50018 Zaragoza, Spain.

jenriccio@unizar.es 